\title{
Permanent Artificial Respiration by Diaphragm Pacemaker in Tetraplegic Children *
}

H. Garrido, M.D., ${ }^{1}$ J. Mazaira, M.D., ${ }^{2}$ P. Gutierrez, M.D., ${ }^{2}$ F. Sebastian, M.D., ${ }^{2}$ J. L. P. Jativa, M.D. ${ }^{2}$ E. M. Serrano, M.D., ${ }^{2}$ J. L. Bermadez, M.D. ${ }^{2}$ and M. A. Ayucar, M.D. ${ }^{2}$

${ }^{1}$ Medical School, University of Cadiz, ${ }^{2}$ Hospital Nacional de Paraplejicos, Toledo, Spain

\section{Summary}

This is a study of two children, aged respectively 10 and 6 years, who sustained a spinal cord injury at the $C 1 \mathrm{C2}$, causing apnoea. After the bilateral implantation of diaphragm pacemakers and periods of conditioning, they were able to adapt to continuous and simultaneous full-time ventilatory support of both diaphragms without any sign of fatigue, for more than 24 and 11 months, respectively.

Key words: Tetraplegia; Children; Pacemaker; Diaphragm; Ventilation.

\section{Introduction}

Permanent artificial ventilation, achieved by electrostimulation of the phrenic nerve by means of the implantation of diaphragm pacemakers in tetraplegic patients with apnoea because of central paralysis of the diaphragm and other respiratory muscles, were reported by Glenn et al. (1972). Permanent ventilation was obtained with respiratory frequencies of 12 to 15 per minute, by high frequency electric stimulation (e.g. electric pulsations of $150 \mathrm{msec}$ with short intervals between each pulsation and thus creating a square wave of $1.3 \mathrm{sec}$ ), and with amplification for each patient to stimulate alternatively one single hemidiaphragm during 12 hours with similar time intervals of rest to avoid fatigue.

Because of the exceptional conditions in young children it was not always possible to obtain permanent ventilation, because of the immaturity of the lungs, thorax and diaphragm, resulting in low current ventilation volumes, especially in the left lung.

After these experiences, and the publication of the results, Glenn initiated electrostimulation of the phrenic nerve (and consequently of the diaphragm),

Requests for reprints: J. Mazaira, Jefe de Servicio de Medicine. Hospital Nacional de Paraplejicos 45071-TOLEDO, SPAIN.

$\star$ Read at the Scientific Meeting of the II Euro-Mediterranean and Ibero-American Congress of Paraplegia, Toledo, April, 1985. 
with continuous artificial lung ventilation, simultaneous for both lungs, without observing any negative effect in the transmission activity in electro-impulses to the nerve and the diaphragm.

Our interest focussed on the results obtained in tetraplegic children with apnoea who, after the implantation of diaphragm pacemakers and with corresponding conditioning periods, were able to adapt themselves to continuous and simultaneous ventilation of both diaphragms without any sign of fatigue.

\section{Material and methods}

Two children, a boy and a girl, aged 10 and 6 years respectively sustained spinal cord lesions at the level of $\mathrm{C} 1-\mathrm{C} 2$ as a result of car accidents. They were studied before the implantation of diaphragm pacemakers, with an interval between the accident and the implantation of pacemakers of one year for the boy and nine months for the girl.

This study is based on radiographic confirmation of immobility of the diaphragm and exploration of the conductivity of the phrenic nerve. (Newson, 1967 \& Glenn et al., 1980). The results are shown in Table 1.

Table 1 Conduction time of the phrenic nerve and potentials of action of the diaphragmatic pacemaker

\begin{tabular}{lrrrrr}
\hline & Age & \multicolumn{2}{c}{$\begin{array}{c}\text { Conduction Time of } \\
\text { Phrenic Nerve }\end{array}$} & \multicolumn{2}{c}{$\begin{array}{c}\text { Potentials of Action of } \\
\text { Diaphragmatic Muscle }\end{array}$} \\
\cline { 3 - 6 } & & Right & Left & Right & Left \\
F.S. & 10 & $8 \mathrm{~ms}$ & $8 \mathrm{~ms}$ & $500 \mu \mathrm{v}$ & $500 \mu \mathrm{v}$ \\
N.M. & 6 & $3.5 \mathrm{~ms}$ & $7.5 \mathrm{~ms}$ & $750 \mu \mathrm{v}$ & $250 \mu \mathrm{v}$ \\
\hline
\end{tabular}

The operative technique for implantation and stimulation of diaphragmatic pacemakers has been described by Glenn et al. (1980).

In both children the electrodes were applied to each of the phrenic nerves in the mediastinum, 2 centimetres above the aortic arch in the 2 nd frontal intercostal space; in the case of the boy the receptor was implanted subcutaneously on the 7 th-8th rib frontolaterally and, in the girl, in the frontosuperior part of the abdominal wall.

The implantation of the electrodes and pacemakers on the left and right sides were carried out with an interval of 15 days.

Two weeks after the second implantation it was possible to begin with the simultaneous pacing of the two hemidiaphragms. It was necessary in the girl to postpone this for 80 days on the left side because there was neuropraxia of the phrenic nerve on that side, but this recovered after the programmed pacing of the phrenic nerve was applied. (Fig. 1).

Before starting the pacing it was necessary to determine the threshold of excitation and the maximum and submaximum intensities of both hemidiaphragms as described in the technique of Glenn et al.

The excitation thresholds and intensities of stimulation are shown in Fig. 2. 


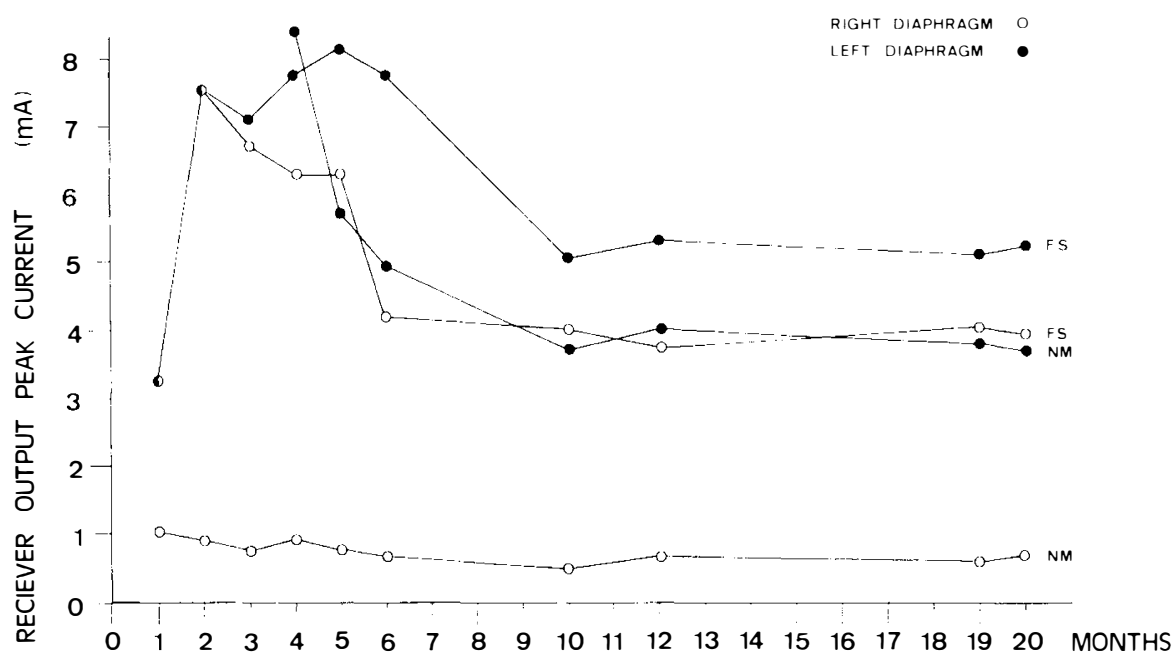

Figure 1. Changes in threshold levels in the two patients are shown during the conditioning period for diaphragmatic function.

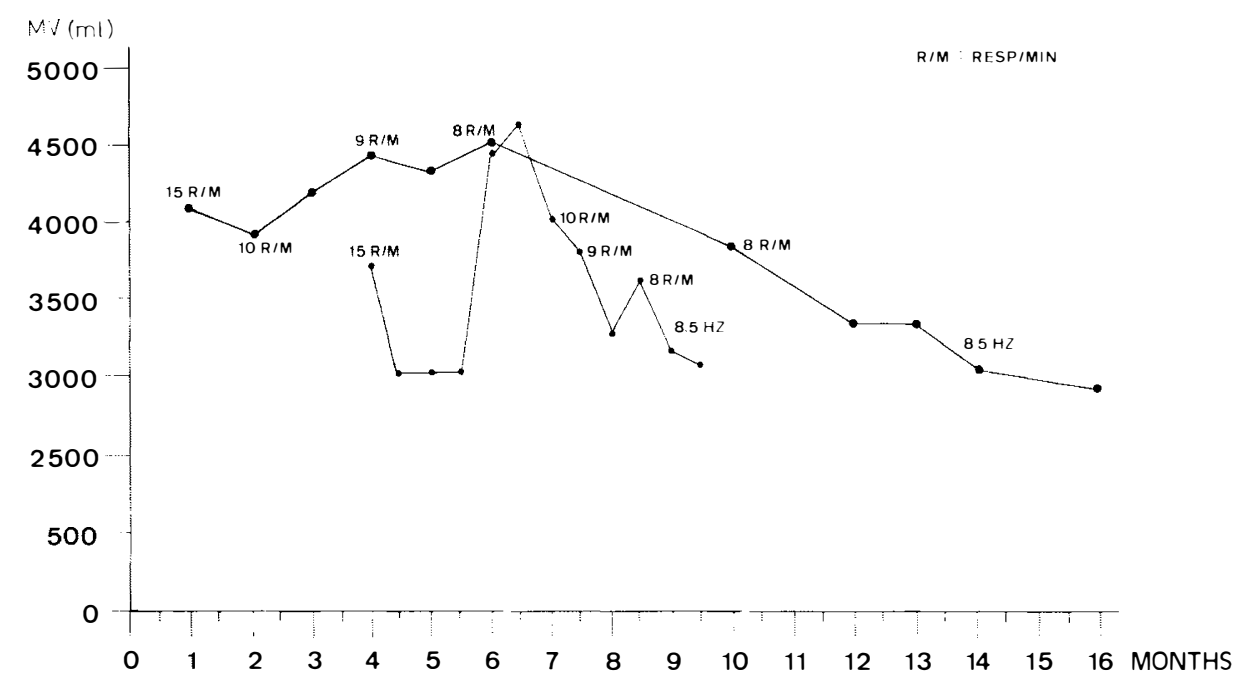

Figure 2. Follow-up of the conditioning period in the two patients. The progressive decrease of respiratory rate and increase of pulse interval in relation of tidal volume are shown.

\section{Conditioning of the Diaphragm}

Once established, the pacing programme for each hemidiaphragm was timed simultaneously 5-10 minutes each hour and during daytime with low frequency pacing $(11 \mathrm{~Hz})$ in a fast rhythm of respiration frequency of 10-15 per minute. The intensity of the electrical stimulation applied was sufficient to obtain a tidal volume for adequate ventilation. Controls were carried out each hour of the tidal volumes and, occasionally, of the blood gases. 
Table 2 Ventilation characteristics for pacemakers in patients

\begin{tabular}{lcccccccc}
\hline Patient & Weight & $\begin{array}{c}\text { Frequency } \\
\mathrm{Hz}\end{array}$ & \multicolumn{2}{c}{$\begin{array}{c}\text { Respiratory } \\
\text { rhythm }\end{array}$} & \multicolumn{2}{c}{$\begin{array}{c}\text { Current } \\
\text { volume }\end{array}$} & \multicolumn{2}{c}{ Time Gasometry } \\
& & & Basic & Actual & ph & PaCo2 & PaO2 \\
\hline $1 .-$ & 32 & $8 \cdot 5$ & 8 resp/min & 384 & 400 & 24 h 7.13 & 36 & 97 \\
$2 .-$ & 22 & $8 \cdot 5$ & 8 resp/min & 260 & 375 & 24 h 7.40 & 31 & 97 \\
\hline
\end{tabular}

The time of hourly pacing was increased every two weeks until a time of 30 minutes was reached; additional increases were programmed giving the same periods of rest to the diaphragm (artifical ventilation with conventional respirator), until 12 hours of pacing had been reached without having observed any sign of diaphragmatic fatigue. This period of conditioning should be extended from 4 to 6 months in infants.

Subsequently the periods of rest between pacings were shortened until a permanent state of ventilation was obtained with this technique (Table 2).

Every 1 or 2 weeks the respiratory frequency was reduced until the period of conditioning became stabilised with 5-8 respirations per minute, thus obtaining the lowest but still sufficient frequency. (Fig. 3).

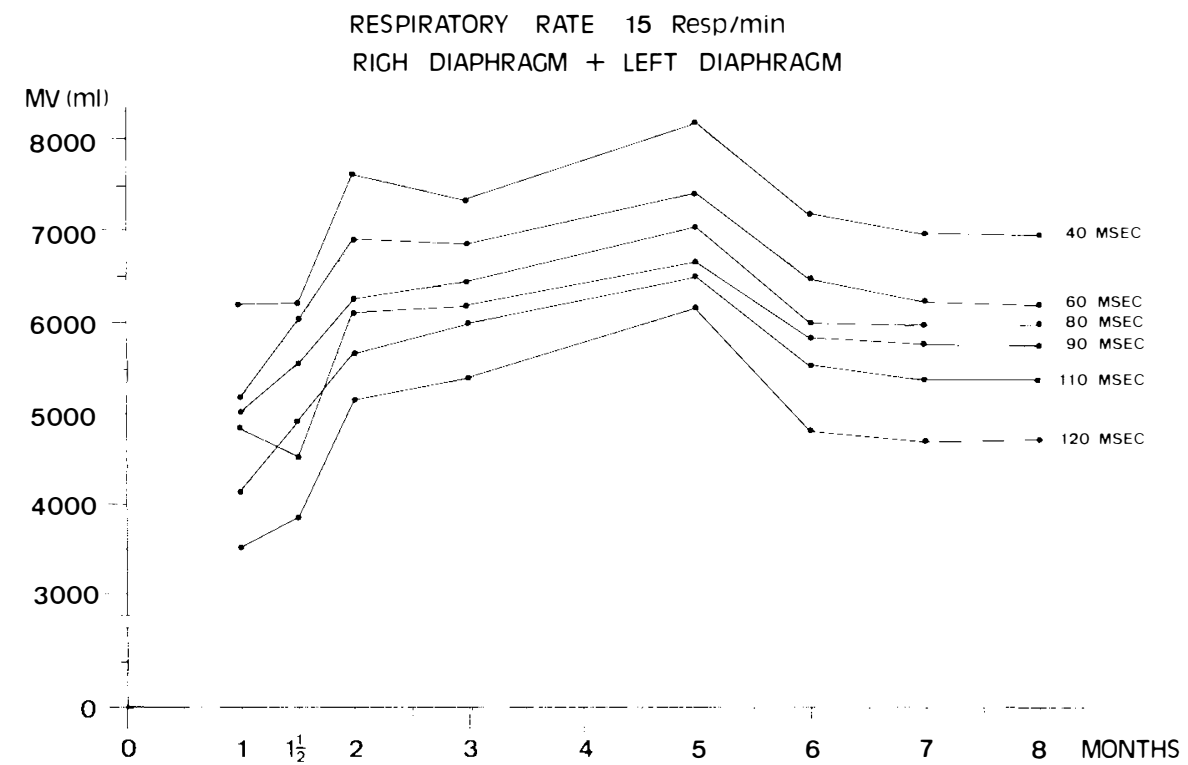

Figure 3. Tidal volume in right and left lungs of the three patients, measured at various pulse intervals to show the increase of the conditioning period.

After 12 hours of pacing the pulse intervals were reduced gradually until, at the end of the treatment, they reached 7.1 to $8.3 \mathrm{~Hz}$ (Table 3).

During the period of conditioning the following controls were carried out at intervals of 15 days.

1. Determination of thresholds by radiological control. 
Table 3 Pacing characteristics

\begin{tabular}{ccrr}
\hline & Pulse frequency & \multicolumn{2}{c}{ intensity } \\
\hline & $\mathrm{Hz}$ & Left & Right \\
$1 .-$ & 8.5 & $7 \mathrm{~mA}$ & $3 \mathrm{~mA}$ \\
$2 .-$ & 8.5 & $1.5 \mathrm{~mA}$ & $3.5 \mathrm{~mA}$ \\
\hline
\end{tabular}

2. Determination of the maximum intensity of pacing by radiological and spirometric controls.

3. Investigation of respiratory volumes (tidal and minute), with pacing of various pulse intervals $(40,60,80,90,110,120,130)-\mathrm{msec}$ by spirometric control.

Both children could manage without the respirator and were therefore being ventilated exclusively by means of the diaphragmatic pacemaker.

Once concluded, the cannula of the tracheotomy tube was removed and substituted by a short teflon tube and a cap.

\section{Results}

The period of conditioning for these children lasted for 7 and 11 months respectively (the more extended period for the second case), due to the complication of the neuropraxia caused by the left phrenic nerve electrode implantation.

At the beginning of this period ventilatory volumes of 15 respirations per minute were reached, decreasing progressively to eight respirations per minute during the 6 th and 7 th month respectively (Fig. 3).

The calibration of the pacing thresholds was irregular in the first case, with a marked increase during the first month, thereafter tending to decrease.

Spirometric exploration of the lungs individually showed increasingly positive evolution until there was stabilisation, and sufficient ventilation with low frequency pacing. A curve is obtained with paradoxical decreases between some of the explorations due to repeated respiratory infections. The differences which were prominent at the beginning of the period of conditioning between high and low frequency pacing, tended to diminish at the end of the period.

\section{Discussion}

Although in the literature there are reports of continuous bilateral pacing of the diaphragm in infants (Radecki, Tomatis, 1976), with apparent success, we are not at all convinced by them, because of limited details of criteria and of documentation dealing with the death of children in the short term.

Glenn et al. (1980) in a group of patients subjected to continuous bilateral electrophrenic pacing, describe a boy of 7 years in whom this method was applied with success for 11 months.

Our two patients, both children, and with daily spirometric controls and revision of the parameters of the electrophrenic pacing every two weeks (with long periods of conditioning between 5 and 11 months respectively), have continuous bilateral pacing with low pulse frequency, $(8.6 \mathrm{~Hz})$ thus maintaining 
the same duration of the train of pulses $(1.3 \mathrm{sec})$ and concluding with respiratory frequencies of 7.8 respirations per minute, in each case, without any deterioration of their general condition and of respiratory sufficiency.

The basic parameters which permit this kind of ventilation are possible in tetraplegic patients since they remain at permanent rest owing to their paralysis.

The doubts that Glenn et al. have expressed about the application of this mode of electrophrenic ventilation in children due to immaturity of the lungs, the thoracic wall or of the diaphragm can definitely be discarded, in our experience, as both children adapted themselves to the period of conditioning and reached levels of sufficent ventilation without mechanical aid.

Although in the adult it would be possible to sum up the advantages of continuous bilateral ventilation versus alternating ventilation due to its efficiency and lack of paradoxical motion on the contralateral side, it is proved that simultaneous pacing produces superior volumes than the sum of the volumes originated by the alternated unilateral pacing.

If these advantages, and many others, are arguments which justify the continuous simultaneous ventilation of both diaphragms in the adult, then in the infant (due to the low tidal volumes that the left lung produces) alternating ventilation is not practicable in many cases; only the simultaneous ventilation of both diaphragms results in adequate ventilation. The parameters however, reveal sporadically occurring episodes of moderate hypercapnia and hypoxia.

It is possible to obtain permanent ventilation without mechanical means with the aid of a tracheostomy tube and obtain a great number of advantages in the treatment of these tetraplegic children, thus achieving family life with possibilities of education and living together in a normal way.

The low pulsation frequencies and the reduction of the respiratory frequency make permanent ventilation possible thanks to a prolonged period of conditioning in the children, thus avoiding fatigue in the diaphragmatic muscles.

It is also important to emphasise that to be able to reach acceptable tidal volumes with low pulsation frequencies it is essential to have a long conditioning period; although high frequency ventilation is sufficient from the start, the low frequencies function only at the end of the long period.

\section{References}

GLENN WWL, M Holcomb WG, MCLANGhlin AJ, et al. 1972 Total ventilatory support in a quadriplegic patient with radio-frequency electrophrenic respiration. New England Journal of Medicine 286.

GlenN WWL, Hogan JF, LAKE JSD, et al. 1984 Ventilatory support by pacing of the conditioned diaphragm in quadriplegia. The New England Journal of Medicine 310:1150-1155.

GLENN WWL, Hogan JF, Phelps ML 1980 Ventilatory support of the quadriplegic patient with respiratory paralysis by diaphragm pacing. Surgical Clinics of North America 5:1055-1078.

Newsom Davis J 1969 Phrenic Nerve Conduction in man. Journal of Neurology, Neurosurgery and Psychiatry 30:420-426.

RAdECKi L, Tomatis LA 1976 Continuous bilateral electrophrenic pacing in an infant with total diaphragmatic paralysis. J. Pediatric 88:967-971. 